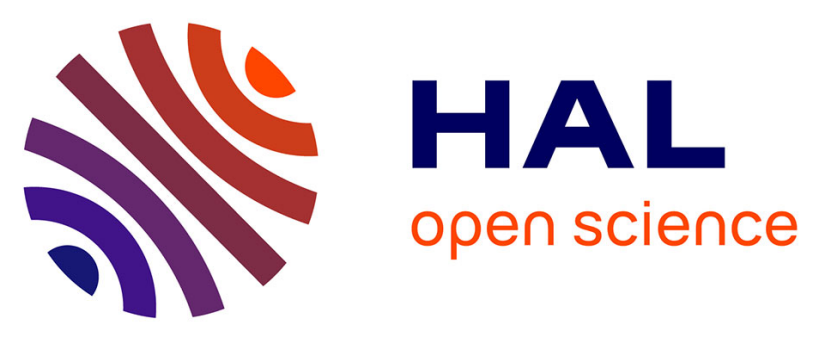

\title{
HOW TO TRAIN TODAY THE TEACHERS OF FRENCH AS A FOREIGN LANGUAGE OF TOMORROW? (The example of an introductory and innovative course in language didactics)
}

Christine Blanchard Rodrigues

\section{To cite this version:}

Christine Blanchard Rodrigues. HOW TO TRAIN TODAY THE TEACHERS OF FRENCH AS A FOREIGN LANGUAGE OF TOMORROW? (The example of an introductory and innovative course in language didactics). $\quad$ /Philological Class, 2021, 26 (3), https://filclass.ru/images/JOURNAL/2021-26-3/3-2021_-200-210.pdf. 10.51762/1FK-2021-26-03-

17. hal-03456086

\section{HAL Id: hal-03456086 \\ https://hal.science/hal-03456086}

Submitted on 29 Nov 2021

HAL is a multi-disciplinary open access archive for the deposit and dissemination of scientific research documents, whether they are published or not. The documents may come from teaching and research institutions in France or abroad, or from public or private research centers.
L'archive ouverte pluridisciplinaire HAL, est destinée au dépôt et à la diffusion de documents scientifiques de niveau recherche, publiés ou non, émanant des établissements d'enseignement et de recherche français ou étrangers, des laboratoires publics ou privés. 


\title{
HOW TO TRAIN TODAY THE TEACHERS OF FRENCH AS A FOREIGN LANGUAGE OF TOMORROW? (The example of an introductory and innovative course in language didactics)
}

\author{
Christine B. Rodrigues \\ Université Clermont Auvergne (Clermont-Ferrand, France) \\ ORCID ID: https://orcid.org/0000-0003-2457-0625
}

\begin{abstract}
A bstract. Training for language teaching profession is a challenge, where innovative practices and digital technology can bring benefits. In this article, we focus on the training of future teachers of French as a foreign language. We present an innovative teaching, mixing a flipped classroom and blended learning approach, for students following an introductory course in language didactics. The aim of this research is to understand how students perceive the language teaching profession before the course, and how they see their development of skills related to their future profession after attending the training. Our approach consists of a text analysis and qualitative analysis of declarative data from participants in this innovative scheme, before the course, and after the course, with a view to ascertaining their appreciation of the development of skills to become a language teacher. We collected the students' responses to surveys given before and after the course in the first semester of the academic year 2020/2021. The first survey, given before the course, dealt with the words associated with teaching, learning and class, as well as the definition of a good language course and the profession of FFL teacher. The second survey, given after the course, concerned the usefulness of the course for the professional objective and a self-evaluation of the skills developed. The analysis shows that the participants have a positive view of the teaching profession, but also describe it in a more traditional way. Results also show that most students felt that the course was useful for their professional life and that they developed several competences related to their definition of the profession.

These results lead to the conclusion that these representations about FFL teachers have to be taken into account during the course, in order to make the students aware of the different facets of the profession.
\end{abstract}

Ke ywords: pre-service teachers; innovation; foreign language teaching; French as a foreign language; flipped classroom; blended learning.

\section{КАК ПОДГОТОВИТЬ СЕГОДНЯ БУДУЩЕГО УЧИТЕЛЯ ФРАНЦУЗСКОГО ЯЗЫКА КАК ИНОСТРАННОГО? (На примере инновационного вводного курса по лингводидактике)}

\author{
Родригес К. Б. \\ Университет Клермон Овернь (Клермон-Ферран, Франция) \\ ORCID ID: https://orcid.org/0000-0003-2457-0625
}

А н н о $m a$ u $и$. Подготовка учителей иностранного языка является непростой задачей, реализация которой сегодня требует обязательного использования инновационных практик и цифровых технологий. Данная статья посвящена проблеме подготовки будущих учителей французского языка как иностранного. Автор представляет инновационный подход в обучении, объединяющий модель перевернутого класса и модель смешанного обучения студентов в рамках вводного курса по лингводидактике. Цель исследования - проанализировать, как студенты воспринимают профессию учителя иностранного языка до прохождения предлагаемого курса и как они видят и осознают развитие своих умений и навыков, относящихся к профессии, после него. Методы исследования заключаются в качественном и количественном анализе данных респондентов, участвовавших в новой программе. Полученные данные были собраны в результате анкетирования студентов в первом семестре 2020-2021 учебного года. Предварительный опрос базировался на выявлении ассоциаций к словам преподавание, учение и класс, а также личном определении таких понятий, как хороший языковой курс и профессия учителя иностранного языка. Опрос после освоения предлагаемого курса был сосредоточен на выявлении пользы самого курса с точки зрения профессиональной цели и самооценки сформированных навыков и умений.

Анализ данных показал, что участники позитивно оценивают профессию учителя и описывают ее с позиции традиционной точки зрения. Результаты также показывают, что большинство студентов увидели пользу предлагаемого курса для своей профессиональной жизни и признали, что в ходе обучения были 
сформированы некоторые необходимые компетенции, относящиеся к будущей профессии. Полученные результаты позволяют заключить, что составленные определения профессии учителя иностранного языка должны быть использованы в ходе курса обучения с тем, чтобы помочь студентам сформировать полное представление о данной профессии.

Ключев ы е слов $a$ : предпрофессиональная подготовка учителей; инновации; обучение иностранному языку; французский язык как иностранный; перевернутый класс; смешанное обучение.

Для иитирования: Родригес, К. Б. Как подготовить сегодня будущего учителя французского языка как иностранного? (На примере инновационного вводного курса по лингводидактике) / К. Б. Родригес. - Текст : непосредственный // Филологический класс. - 2021. - Т. 26, № 3. - С. 200210. - DOI: 10.51762/1FK-2021-26-03-17.

Introduction. La formation aux métiers de l'enseignement des langues représente un enjeu, par exemple en langue de spécialité où une "véritable formation préalable» est nécessaire [Faure 2014]. Le numérique (Tice pour le domaine de la didactique des langues) peut avoir un rôle dans ce contexte, tenant compte de l'évolution constante de ces «toujours nouvelles technologies» [Perriault 2002]. Dans cet article, nous décrivons la scénarisation mise en place pour un enseignement initiant à la didactique des langues et plus particulièrement du Français Langue Étrangère (FLE). Nous nous interrogeons en particulier sur l'idée que se font les étudiants inscrits dans cette formation du métier d'enseignant de langue étrangère, et sur leur auto-évaluation de compétences développées à l'issue du cours.

\section{Méthodes de recherche.}

Contexte de l'étude. Notre étude est basée sur une innovation mise en place dans le cadre d'une option initiant à la didactique du FLE, proposée aux étudiants de l'Université Clermont Auvergne en France (Département de linguistique, UFR Lettres, Culture et Sciences Humaines). Ce public est en troisième année de Licence, dans les formations spécialisées en Sciences du Langage, en Lettres, et Langues Étrangères. Il comprend également des étudiants inscrits en Master FLE n'ayant pas validé cette option préalablement à leur inscription en $2^{\text {ème }}$ cycle, à l'université Clermont Auvergne. L'option FLE permet aux étudiants d'être sensibilisés aux problèmes linguistiques, communicatifs, culturels et pédagogiques que pose l'enseignement/apprentissage des langues étrangères.
For citation: Rodrigues, Ch. B. (2021). How to Train Today the Teachers of French as a Foreign Language of Tomorrow? (The example of an introductory and innovative course in language didactics). In Philological Class. Vol. 26. No. 3, pp. 200-210. DOI: 10.51762/1FK-2021-26-03-17.

Une restructuration des cours, dans le cadre du projet Miel-FLES ${ }^{1}$, a été mise en œuvre avec l'objectif de dynamiser les enseignements de cette option. Le terrain d'expérimentation choisi concernait deux enseignements : Introduction à la didactique des langues (IDIL), au semestre 5, et Problèmes pédagogiques et culturels du FLE (PPEC), au semestre 6.

Public visé. Les étudiants qui suivent l'option FLE n'ont en majorité pas d'expérience professionnelle dans l'enseignement, à fortiori dans la formation aux langues étrangères. Pourtant, au sortir de la Licence, une partie d'entre eux est amenée à réaliser des activités d'enseignement, tels que des séjours en tant qu'assistant de langue, ou des stages d'enseignement au primaire ou au secondaire.

De plus, le contenu des enseignements en lien avec la didactique des langues et du FLE n'est pas abordé avant l'année 3 de Licence, et représente généralement une découverte pour ce public. Bien que certains étudiants aient suivi des cours en lien avec la linguistique (par exemple, en morphosyntaxe, en phonétique/phonologie, en traduction), ces différentes spécialités n'étaient pas spécifiquement abordées à l'aune de l'enseignement du FLE, et ne leur permettait

\footnotetext{
${ }^{1}$ Modules d'initiation à l'Enseignement des Langues Français Langue Étrangère et Seconde), UFR Lettres, Culture et Sciences Humaines (LCSH) et Laboratoire de Recherche sur le Langage (LRL, EA 999), Université Clermont Auvergne Projet Learn'in Auvergne (Programme transverse du projet CAP 20-25, I-Site, UCA): https://cap2025.fr/formation/learnin-auvergne/ thematiques-des-projets/miel-module-dinitiation-alenseignement-des-langues.
} 
donc pas de conduire une réflexion sur leur métier qu'ils envisagent de pratiquer (connaissances sur l'histoire de la didactique des langues, besoins du public non-natif, prise en compte de langue-culture, etc.).

L'enjeu était donc d'initier pendant deux semestres ce public à la discipline, tout en le préparant à l'agir professionnel, cela tenant compte du temps limité (deux heures par semaine ne permettant pas, par exemple, de réaliser un stage) et en mettant en œuvre une approche innovante, favorisant notamment l'autonomie des étudiants via l'usage d'outils numériques et de modalités nouvelles.

Approche scientifique pour le scénario pédagogique. Pour la mise en œuvre du dispositif, nous avons procédé suivant deux approches, largement reconnues dans la littérature comme favorisant l'apprentissage. La première, en classe inversée [Bergmann \& Sams 2012; Lebrun \& Lecoq 2015; Faillet, 2014], a été adoptée pour les contenus théoriques afin de rendre les étudiants plus actifs et ne pas limiter la formation théorique à une transmission des savoirs. La deuxième consiste en la formation hybride [Garrison \& Kanuka 2004; Albero \& Safourcade 2014; Bryan \& Volchenkova 2016; Nissen 2011]. L'adoption de ces deux approches vise à rendre les étudiants plus autonomes et plus actifs [Mucchielli 2008;
Rege Colet \& Berthiaume 2013], et à créer une situation de co-construction des savoirs [Vygotsky 1978; Jacoby \& Ochs 1995]. Cela permet aussi de ne pas se limiter à un enseignement directif avec transmission des savoirs [Lebrun, Gilson \& Goffinet 2016].

Mise en œuvre. La mise en œuvre est similaire pour les deux unités d'enseignement concernées Une première séance est consacrée à la présentation du dispositif, afin d'expliquer aux étudiants les contenus qui seront abordés ainsi que les modalités classe inversée et hybridation. Sont également réalisées deux tâches: une activité brise-glace et un questionnaire en ligne (Wooclap), pour vérifier la bonne compréhension des principes du cours par les participants. Au cours des cinq semaines suivantes, les étudiants visionnent à distance et en asynchrone une capsule vidéo de cours par semaine avant la séance, suivie d'un quiz vérifiant leur compréhension des contenus. En fonction des résultats obtenus, ils sélectionnent en classe l'un des deux parcours proposé (parcours I si le quiz n'est pas réussi, parcours 2 si le quiz est réussi).

En ce qui concerne le module IDIL, les cours portent principalement sur la langue à enseigner, les contenus, et les tâches de grammaire et de communication (tableau 1).

Tableau 1. Thèmes et contenus théoriques abordés dans le module IDIL

\begin{tabular}{|l|l|}
\hline \multicolumn{1}{|c|}{ Thème } & \multicolumn{1}{c|}{ Contenu présenté dans le cours } \\
\hline Quelle langue enseigner? & 1) La langue, un système et un outil de communication \\
\cline { 2 - 3 } & 2) La question de la norme et de la variation \\
\hline Quels contenus enseigner? & 3) Les référentiels en didactique des langues \\
\hline Quelles activités d'apprentissage proposer? & $\begin{array}{l}\text { 4) Activités d'apprentissage : les exercices de } \\
\text { grammaire }\end{array}$ \\
\cline { 2 - 3 } & $\begin{array}{l}\text { 5) Activités d'apprentissage : les tâches de } \\
\text { communication }\end{array}$ \\
\hline
\end{tabular}

Pour le module PPEC, les contenus concernent les publics et l'enseignement / apprentissage de la culture (tableau 2).

Tableau 2. Thèmes et contenus théoriques abordés dans le module PPEC

\begin{tabular}{|l|l|}
\hline \multicolumn{1}{|c|}{ Thème } & \multicolumn{1}{c|}{ Contenu présenté dans le cours } \\
\hline $\begin{array}{l}\text { A quel public peut-on être amené à enseigner le } \\
\text { FLE? }\end{array}$ & 1) Les publics-cibles dans l'enseignement du FLE \\
\hline Comment enseigner une langue étrangère? & 2) Particularités de l'enseignement des langues \\
\hline Quelle culture enseigner? & $\begin{array}{l}\text { 3) La notion de culture dans l'enseignement du FLE } \\
\text { 4) Enseigner et apprendre une langue-culture }\end{array}$ \\
\cline { 2 - 2 } & $\begin{array}{l}\text { 5) Démarche interculturelle dans l'enseignement- } \\
\text { apprentissage du FLE }\end{array}$ \\
\hline
\end{tabular}

Au cours des quatre semaines suivantes, les étudiants travaillent par petits groupes pour concevoir des tâches d'apprentissage du
FLE (description d'un public et ses besoins, écriture d'une consigne et de la tâche, justification de la proposition), permettant 
une application concrète des savoirs. Après le dépôt sur une base de données de leurs propositions, chaque étudiant dispose de deux semaines pour procéder à une évaluation des pairs, en commentant l'une des tâches créées par un autre groupe.

Cette partie du cours est en présentiel une semaine sur deux. Les participants utilisent des espaces de groupe (Teams), incluant un outil d'écriture collaborative (Word on line) pour concevoir ces activités en groupe, et la plateforme Moodle de l'université pour déposer leurs propositions de tâches et réaliser l'évaluation individuelle des pairs. Ils sont accompagnés suivant les modalités hybrides par l'enseignante, pendant la classe une semaine sur deux, et à distance et en asynchrone grâce aux outils de la CMO intégrés à Moodle et Teams et à la fonction commentaires de Word.

Pour l'unité d'enseignement IDIL, les étudiants créent une seule tâche (tableau 3). Ce cours est en effet une véritable initiation à la didactique des langues et la création d'une tâche, accompagnée de sa consigne et de sa justification, occupe pleinement le temps consacré à cette réalisation en mode hybride.

Tableau 3. Thèmes et contenus de la partie travaux dirigés du cours IDIL

\begin{tabular}{|l|l|}
\hline \multicolumn{1}{|c|}{ Thèmes } & \multicolumn{1}{|c|}{ Contenus } \\
\hline $\begin{array}{l}\text { 1) Définir des compétences langagières et } \\
\text { communicatives à développer pour un public } \\
\text { d'apprenants }\end{array}$ & $\begin{array}{l}\text { Manipulation du CECRL pour situer des exemples de } \\
\text { tâches proposées dans des manuels de FLE }\end{array}$ \\
\hline 2) Élaborer une tâche en FLE & $\begin{array}{l}\text { Élaboration de la consigne et du contenu de la tâche } \\
\text { Rédaction de la justification s'appuyant sur le CECRL } \\
\text { et sur le cours }\end{array}$ \\
\hline 3) Évaluer les pairs & $\begin{array}{l}\text { Création d'une grille d'évaluation critériée d'une tâche } \\
\text { Rédaction d'un commentaire évaluant les } \\
\text { propositions de tâches }\end{array}$ \\
\hline
\end{tabular}

Pour compléter ce processus et développer les compétences des étudiants, une fiche d'activités comprenant plusieurs tâches est demandée dans le cours PPEC (tableau 4). Les participants doivent imaginer une série de tâches et non plus seulement une tâche isolée.

Tableau 4. Thèmes et contenus de la partie travaux dirigés du cours PPEC

\begin{tabular}{|l|l|}
\hline \multicolumn{1}{|c|}{ Thèmes } & \multicolumn{1}{|c|}{ Contenus } \\
\hline $\begin{array}{l}\text { 1) Définir des compétences langagières et } \\
\text { communicatives à développer pour un public } \\
\text { d'apprenants }\end{array}$ & $\begin{array}{l}\text { Manipulation du CECRL pour situer des exemples de } \\
\text { tâches proposées dans des manuels de FLE } \\
\text { Réflexion sur la fiche d'activités qui sera élaborée }\end{array}$ \\
\hline 2) Élaborer une fiche d'activités en FLE & $\begin{array}{l}\text { Élaboration des consignes et des contenus de la tâche } \\
\text { Rédaction de la justification s'appuyant sur le CECRL } \\
\text { et sur le cours }\end{array}$ \\
\hline 3) Évaluer les pairs & $\begin{array}{l}\text { Création d'une grille d'évaluation critériée d'une fiche } \\
\text { d'activités } \\
\text { Rédaction d'un commentaire évaluant les } \\
\text { propositions de fiches d'activités }\end{array}$ \\
\hline
\end{tabular}

Cette scénarisation pédagogique, qui rend le parcours d'initiation à la didactique et ses objectifs plus clairs, répond aux caractéristiques que nous avons évoquées sur les approches classe inversée et hybridation :

- Appropriation des contenus par les capsules vidéo, les quiz et les tâches, adaptée au contexte actuel des Tice, et permettant de bénéficier des temps de présence en cours pour revoir les contenus et les appliquer, et favorisant un apprentissage actif et autonome, adaptée au contexte actuel du numérique.

- Mobilisation des connaissances dans des tâches créatives et d'évaluation, utiles pour le métier d'enseignant.

- Soutien à la co-construction des savoirs par les tâches collaboratives, favorisant les échanges en présentiel et à distance, en synchrone et en asynchrone et un accompagnement tutoral plus ciblé et adapté.

- Formation aux outils numériques et à de nouvelles modalités d'enseignement, également utiles à l'enseignant de langue du futur.

Par ailleurs, les étudiants développent des compétences professionnelles puisqu'ils doivent définir les besoins et caractéristiques 
du public choisi, décrire les objectifs, se baser sur le Cadre Européen commun de référence pour les Langues [Conseil de l'Europe 2001], concevoir une tâche accompagnée d'une consigne, et commenter le travail de leurs pairs en faisant preuve de bienveillance [Blanchard et al. 2021].

Objectif de la recherche. L'objectif de la recherche est de comprendre quelles idées les futurs enseignants de langues étrangères se font du métier avant le cours, et d'observer comment, après avoir suivi la formation, ces étudiants voient leur développement de compétences en lien avec leur futur métier et leur appréciation du dispositif en tant que nouvelle méthode d'enseignementapprentissage.

Nous avons sélectionné les réponses des participants à l'étude en lien avec leurs conceptions du métier d'enseignant de langue avant le cours (questionnaire 1), ainsi que celles représentant les compétences qu'ils pensent avoir développé pour leur projet professionnel (questionnaire 2).

Données recueillies. Pour la présente étude, nous avons recueilli les réponses des étudiants au premier semestre de l'année universitaire 2020/2021 à deux questionnaires distribués lors de la formation IDIL. Le premier questionnaire (26 répondants) a été dispensé depuis la plateforme en début de formation, et comprenait questions ouvertes et deux questions fermées (questionnaire 1). Pour cette analyse, nous avons observé les réponses, concernant cinq questions (en retenant pour les occurrences apparaissant au moins deux fois dans le corpus en ce qui concerne les trois premières questions) :

- Quels mots-clés associez-vous à

«Enseignement »?

- Quels mots-clés associez-vous à

«Apprentissage»?

- Quels mots-clés associez-vous à

«Classe »?
- Qu'est-ce qu'un «bon » cours de langue étrangère selon vous?

De manière générale, comment concevez-vous le métier d'enseignant de FLE ?

Nous avons également relevé les réponses au questionnaire proposé après la formation IDIL (22 répondants), comprenant onze questions ouvertes et vingt-neuf questions fermées (questionnaire 2). Nous avons considéré les réponses aux questions concernant l'utilité de l'enseignement suivi pour la vie professionnelle et les compétences développées pendant le cours. Les réponses aux deux questionnaires ont été exportées depuis la plateforme Moodle au format xls pour l'analyse.

Approche scientifique. Pour la présente étude, nous avons adopté une méthodologie d'analyse textuelle de type traitement statistique avec comptage de mots, pour relever les éléments les plus cités par les participants pour ce qui est de leurs descriptions de leur futur métier. Notre démarche consiste également en l'analyse qualitative de données déclaratives des participants à ce dispositif innovant, en vue de connaitre leur conception d'un bon cours de langue étrangère et du métier d'enseignant de FLE avant la formation, et leur appréciation du développement de compétences pour devenir enseignant de langue après le cours.

Résultats et discussion. L'analyse textuelle des occurrences nous permet de relever ce que les étudiants associent aux termes enseignement, apprentissage et classe. Une diversité de mots sont liés à l'enseignement: on relèvera notamment passion ( 2 occurrences) et partage (4 occurrences), qui semblent montrer des représentations dynamiques du rôle de l'enseignant. Toutefois quelques termes relèvent d'une conception plus traditionnelle de l'enseignement : transmettre et transmission sont majoritaires dans les réponses des participants (Figure 1). 


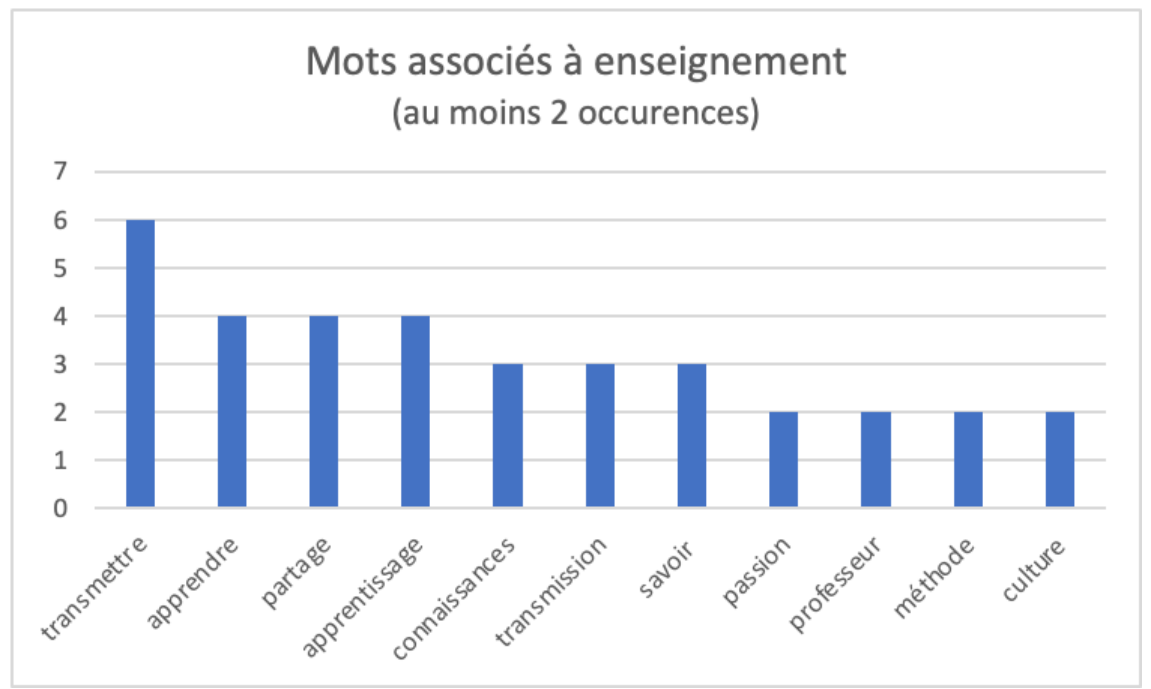

Fig. 1. Mots les plus fréquemment associés à « enseignement »

De même, le concept d'apprentissage est majoritairement relié pour ces étudiants à des termes proches tels que apprendre, savoir et connaissance(s) (Figure 2). Les participants voient également dans l'apprentissage la capacité de progrès (améliorer, évolution). On note également l'usage du terme élève, bien qu'en didactique des langues est plutôt fait usage du mot apprenant (qui lui n'apparait pas dans les résultats).

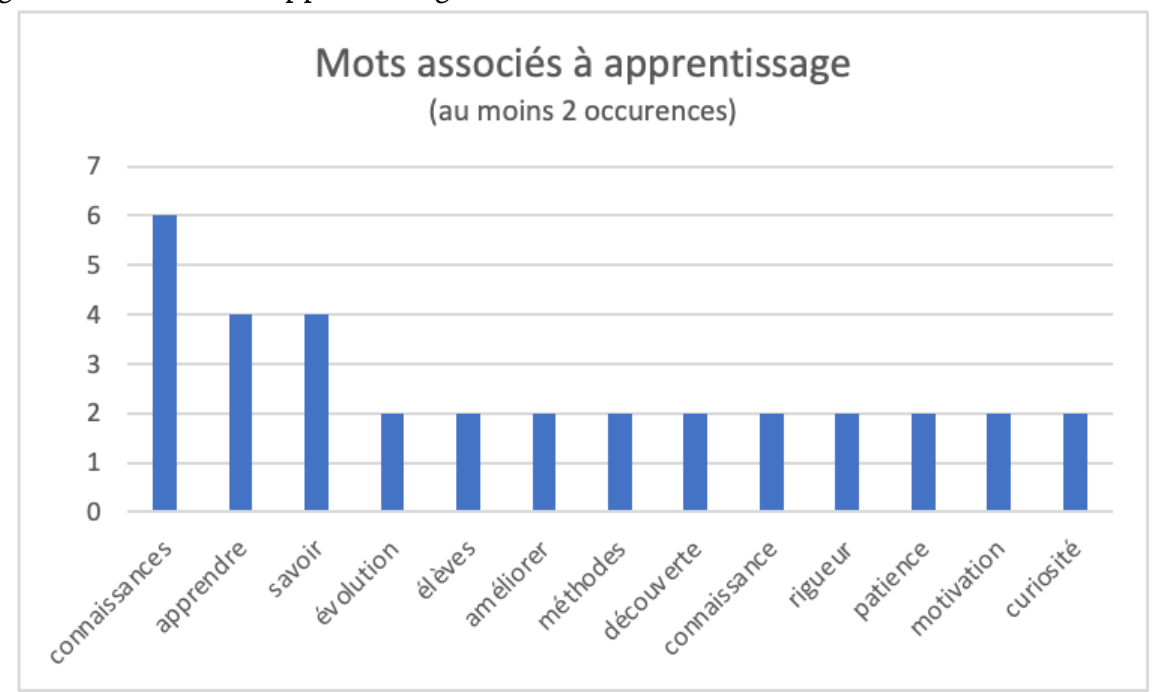

Fig. 2. Mots les plus fréquemment associés à « apprentissage »

Toutefois, lorsque l'on interroge sur les mots associés à classe, certains participants citent apprenant et enseignant (Figure 3). Il est également intéressant de noter qu'une vision plutôt dynamique apparait dans les résultats (échanges, communauté, diversité, entraide et ensemble). 


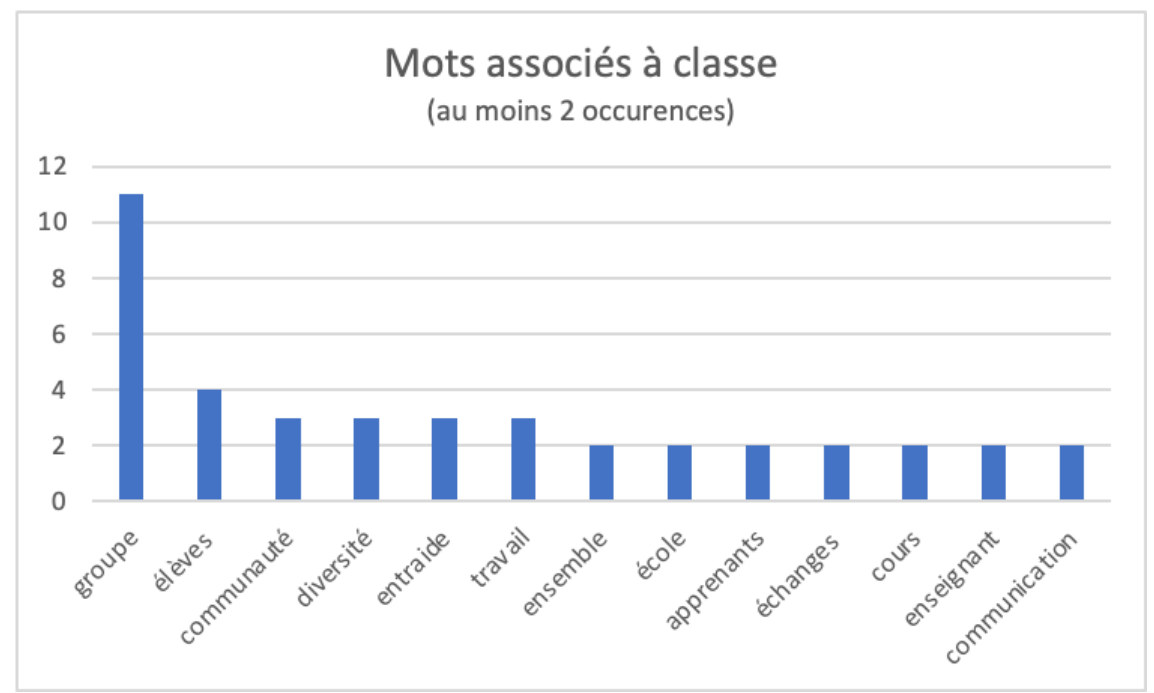

Fig. 3. Mots les plus fréquemment associés à « classe »

Cette idée d'un dynamisme créé par les interactions en classe de langue se retrouve dans les réponses à la question «Qu'est-ce qu'un «bon » cours de langue étrangère selon vous?» :

- «Un cours qui fait participer les élèves, et pas seulement le professeur. Un cours de langue étrangère, c'est avant tout un échange. »

- «Un cours dans lequel les apprenants, parce qu'ils sont intéressés par le cours, participent. »

- «Un cours interactif»

- «Un cours qui implique les élèves, les intéresse et les fait participer (y compris en les faisant échanger entre eux). Le cours doit donc revêtir, au moins en partie, un aspect ludique. »

- «Un cours avec un climat de confiance entre les élèves et le professeur, où la participation est encouragée avec le respect des uns et des autres.»

- «Un bon cours de langue c'est lorsque tout le monde osent participer sans avoir peur d'échouer.»

La pratique de l'oral est également mise en avant :

- «Se concentrer plus sur l'oral que l'écrit. »

- «Une ambiance active, des activités que tout le monde peut y participer, la pratique de l'oral. »

- «Selon moi, un «bon» cours de langue étrangère est de pouvoir faire parler les élèves sur un sujet. »

- «Un cours où l'on apprend du vocabulaire, de la conjugaison, où l'on écoute des professeurs ou des productions orales pour améliorer sa compréhension. » « inter-actif, méthodique, ryhtmé »

- « un cours ludique avec une communication orale importante entre prof et élèves mais aussi les élèves entre eux. "

- «Un bon cours de langue étrangère est un cours dans lequel les élèves participent, posent des question si ils en ont. »

Les répondants notent également l'importance du rôle de l'enseignant dans un «bon» cours de langue, en particulier, un enseignement qui doit donner envie d'apprendre, être bien préparé, et facilitateur des apprentissages :

- «Un cours dont le professeur arrive à captivl'élève et à lui donner l'envie d'apprendre. »

- «Il faut avoir un plan concret, le prof doit bien faire la préparation avant les cours. Il faut bien comprendre la situation des étudiants.Dans les cours, il faut bien expliquer. »

- «Selon moi, c'est quand le professeur est dynamique et passionné car l'amour se transmet automatiquement. De plus, les langues étrangères sont souvent appelées langues vivantes donc leur apprentissage nécessite un certain enthousiasme. »

- «Un cours qui me donne envie d'apprendre, et dans lequel je suis à l'aise. »

- «Un cours intéressant et actif, ou chacun se sent concerné. »

- «Un cours clair et facile à suivre qui facilite l'apprentissage de la langue à ses apprenants. » 
- «C'est un cours vivant, structuré et surtout avec un bon enseignant qui motive l'élève à apprendre. »

- «Un cours organisé avec le moins d'improvisation possible (sauf en cas d'imprévu où l'improvisation se révèle inévitable). »

- «Un cours où les élèves apprennent quelque chose d'utile, qui peut leur servir, qui les intéresse, qui ne soit pas déconnecté de la réalité, qui soit un minimum personnalisé au groupe apprenant. »

- «Un cours qui s'adapte aux lacunes et aux atouts individuels de chaque élève. »

Enfin, l'un des participants souligne limportance du numérique :

- «Un cours accessible sur internet (ENT).».

Pour les répondants à la question suivante, le métier d'enseignant de FLE est conçu comme une activité donnant envie d'apprendre à l'apprenant :

- « Je conçois le métier d'enseignant de fle, comme n'importe quel enseignant de langue, il doit être motivé et donner envie aux élèves d'apprendre cette langue. »

- « Enseigner une langue, FLE ou non, doit aussi intéresser les élèves et leur permettre d'apprendre selon la méthodologie la plus efficace possible et leur donner les moyens et la motivation de continuer d'apprendre par euxmêmes après la fin des cours (Exemple : en leur faisant découvrir du contenu culturel en français susceptibles de les intéresser). »

- «Donner envie d'apprendre».

C'est aussi un métier considéré comme difficile et gratifiant à la fois, qui permet de découvrir et d'apprendre :

- «Ce métier est aussi différent du métier d'enseignant de langues à un public français car il faut que celui-ci puisse s'adapter aux méthodes d'apprentissage du pays où il enseigne. »

- «C'est un métier de découverte (si on enseigne dans un autre pays), et ça doit être très enrichissant. »

- «En perpétuel apprentissage. On n'enseigne pas comme il y a 50 ans, et on enseignera peut-être différemment dans ioans. On apprend de ses erreurs, ses réussites, ses élèves, et on cherche à s'améliorer. Gratifiant, peut-être difficile pour certains. »

- «Comme quelque chose à double sens : bien que le professeur de FLE transmet plus à ses élèves que ses élèves ne lui transmettent, il doit savoir apprendre d'eux, apprendre de leurs cultures et langues, comprendre leurs difficultés, etc. »

- «Comme tout autre métier d'enseignant de langue. Difficile mais gratifiant pour l'enseignant, ainsi que pour les élèves s'ils s'investissent. »

Pour les répondants, le métier d'enseignant consiste aussi à faire découvrir une culture :

- « Etre enseignant de FLE, c'est partager une culture qui est étrangère à l'étudiant. C'est pouvoir lui permettre de découvrir une langue et sa culture, et de pouvoir se faire comprendre en Français. »

- «Comme pour les autres langues, c'est transmettre une nouvelle langue avec la culture qui lui est liée. »

- «C'est pour moi un métier où on transmet sa culture, notamment sa langue natale. »

- «Comme un moyen de partage de la culture et de la langue française. »

- «Je le conçois comme une ouverture à d'autres cultures car les apprenants sont tous différents et ils apportent leur richesse culturelle. C'est aussi l'opportunité pour l'apprenant de s'ouvrir à une autre langue et culture. »

- «De manière générale, je conçois le métier d'enseignant de FLE comme une passerelle d'une langue vers une autre, permettant aux élèves de s'ouvrir à une nouvelle culture.»

Les verbatims font également apparaitre l'idée qu'un enseignant de FLE est amené à enseigner à l'étranger, et qu'il s'agit en général d'un natif:

- «Le métier d'enseignant de FLE est riche de cultures étrangères. En effet, le professeur étudie dans de multiples pays étrangers sa langue maternelle. »

- «Je conçois le métier d'enseignant de FLE avant tout comme une chance pour les français. En effet, ce métier permet de marier l'enseignement et les voyages, ainsi, je pense que la majorité des professeurs de FLE sont des passionnés et vont concevoir et s'adapter à leur métier à leur propre manière. »

- «C'est un enseignement particulier puisqu'il s'agit en général d'enseigner sa langue maternelle. Le professeur doit enseigner sa discipline et la culture associée tout en se mettant à la place des apprenants pour les accompagner le mieux possible. » 
Deux étudiants ont par ailleurs répondu ne pas savoir répondre à cette question. Bien qu'engagés dans un parcours pouvant conduire au métier d'enseignant de FLE, profession, ils ne parviennent pas à se représenter cette profession.
Dans le deuxième questionnaire, il a été demandé aux étudiants si les enseignements du cours leur ont semblé utiles pour leur vie professionnelle. Une grande majorité des participants est tout à fait d'accord (64\%) ou plutôt d'accord (4\%) avec cette utilité (Fig. 4).

\section{CES ENSEIGNEMENTS VOUS SEMBLENT UTILES POUR LA VIE PROFESSIONNELLE ?}

- Tout à fait d'accord @ Plutôt d'accord — Plutôt pas d'accord — Pas du tout d'accord

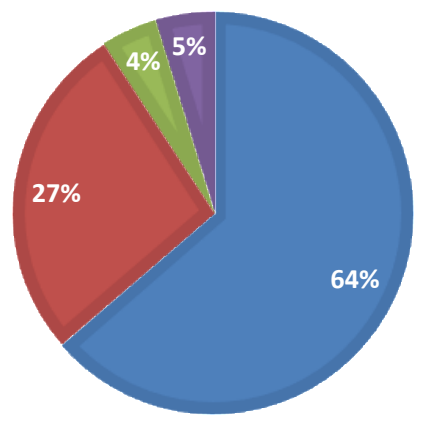

Fig. 4. Réponses des étudiants concernant l'utilité des enseignements pour la vie professionnelle

Les participants ont également évalué les compétences qu'ils pensaient avoir développé à la suite du cours. Les résultats montrent que ces compétences sont en lien avec le futur rôle d'enseignant de langue: organisation, autonomie, prise de décision, communication, collaboration (Fig. 5). Un étudiant a également ajouté la capacité d'imagination.

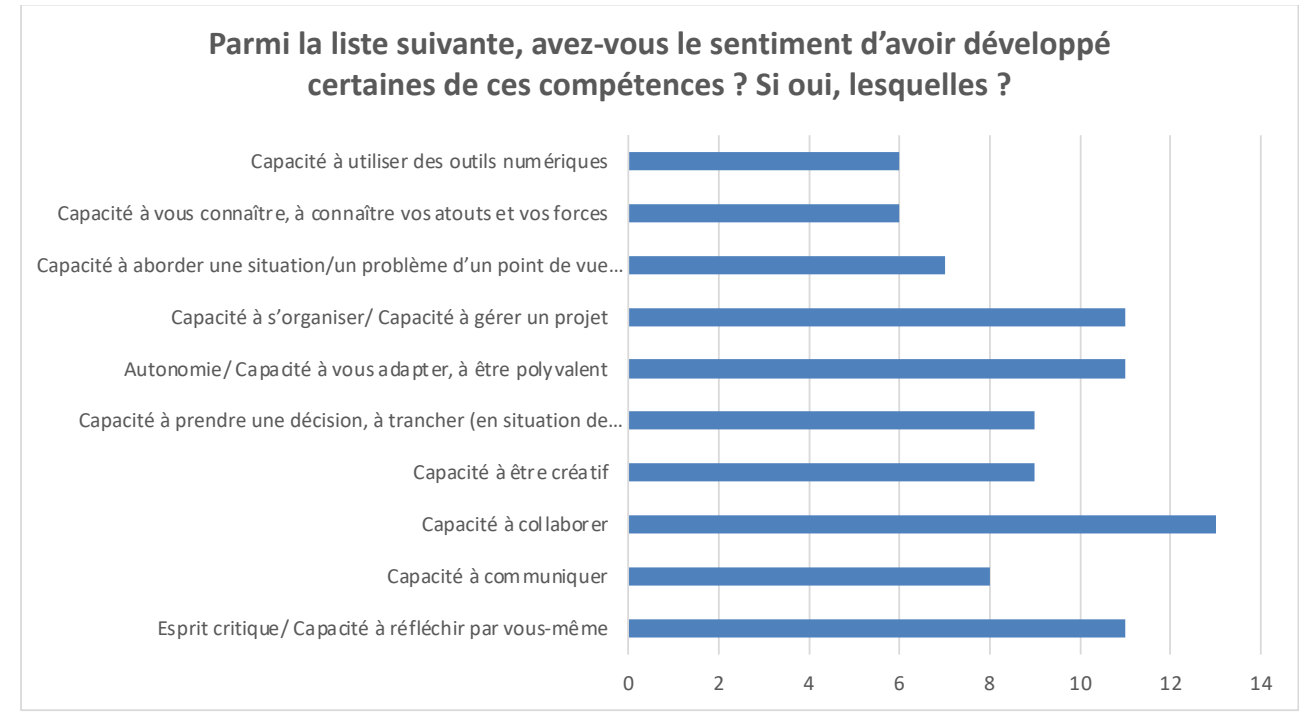

Fig. 5. Réponses des étudiants concernant leur développement de compétences

Dans les commentaires libres, plusieurs étudiants soulignent ces compétences :

- «Ces enseignements nous ont permis de nous perfectionner et de perfectionner notre niveau de langue. surtout pour les étudiants qui ont décidés d'enseigner le 
Français langue étrangère. »

- «J'ai beaucoup aimé ces cours et aussi la tâche à rendre ce fut un plaisir de travailler dessus, ce qui nous pousse à avoir de l'imagination et un esprit créatif. »

- «Ces enseignements ont été très utiles pour mon projet professionnel pour la suite de mes études et travail avec le FLE. »

Le dispositif innovant mis en œuvre semble ainsi avoir un impact favorable sur le développement de compétences relevées comme essentielles pour l'enseignant du futur.

Conclusion. S'ils ont une vision plutôt positive du métier d'enseignant de FLE (importance de la profession, mission enrichissante, dynamisme), les étudiants futurs enseignants peuvent aussi se représenter cette profession de manière plus traditionnelle (acquisition de savoirs) ou ne recouvrant pas l'ensemble du domaine (accent mis sur l'oral et la culture, représentation d'un métier plutôt réservé aux natifs). Le cours proposé semble répondre à ces points, par les sujets abordés et la manière de présenter les contenus (chaque capsule vidéo partant d'idées reçues sur la didactique des langues). En complément, le cours semble avoir aidé les étudiants à développer des compétences qu'ils ont euxmêmes relevé comme essentielles au métier (organisation, créativité, communication).

Cette étude nous semble offrir deux perspectives, l'une en formation, et l'autre sur un axe plus scientifique. Il s'agira tout d'abord de tenir compte des représentations relevées dans cette recherche, pour faire prendre conscience aux étudiants pendant les cours des différentes facettes du métier. Dans une orientation recherche, une nouvelle étude pourra être menée auprès d'un nouveau public intégrant l'option FLE, pour vérifier l'évolution des représentations, en dispensant le questionnaire avant et après la formation (cette étude n'ayant pas pu être menée au préalable en raison du confinement en France en fin d'année 2020), ce qui nous permettra aussi de mesurer plus finement les liens entre le dispositif innovant mis en œuvre et ces représentations.

\section{Références}

Albero, B. \& Safourcade, S. (2014). Compétences et formation à distance : des prescriptions du dispositif aux attitudes des étudiants Enquête exploratoire en école d'ingénieur. In Distances et Médiations des Savoirs, CNED-Centre national d'enseignement à distance. No. 6. URL : http://journals.openedition.org/dms/698.

Bergmann, J. \& Sams, A. (2012). Flip your classroom: Reach every student in every class every day. Eugene, Or, International Society for Technology in Education.

Blanchard, C., Foucher, A.-L. \& Chabanal, D. (2021). Pedagogical innovation in higher education: development of professional skills of pre-service foreign language teachers. In Eurocall. 26-27 August.

Bryan, A. \& Volchenkova, K. N. (2016). Blended Learning: Definition, Models, Implications for Higher Education. In Bulletin of the South Ural State University. Educational Sciences. No. 8 (2), pp. 4-30.

Council of Europe (2001). Common European Framework of Reference for Languages: Learning, teaching, assessment. Cambridge University Press.

Faillet, V. (2014). La pédagogie inversée: Recherche sur la pratique de la classe inversée. In Sciences et Technologies de l'Information et de la Communication pour l'Éducation et la Formation. No. 21 (1), pp. 651-665. URL: https://doi.org/10.3406/stice.2014.1115.

Faure, P. (2014). Enjeux d'une professionnalisation de la formation des enseignants de langue(s) de spécialité: exemples de l'anglais et du français de la médecine. Recherche et pratiques pédagogiques en langues de spécialité. In Cahiers de l'Apliut, Vol. XXXIII. No. 1, pp. 50-65. URL: https://doi.org/10.4000/apliut.4170.

Garrison, D. R. \& Kanuka, H. (2004). Blended Learning: Uncovering its Transformative Potential in Higher Education. In The internet and higher education. No. 7 (2), pp. 95-105.

Jacoby, S. \& Ochs E. (1995). Co-Construction: An Introduction. In Research on Language and Social Interaction. No. 28 (3), pp. 171-183.

Kirkpatrick, D. L. \& Kirkpatrick, J. D. (2010). Evaluating Training Programs: The Four Levels (ReadHowYouWant ed., set in 16 pt. Verdana, complete, unabridged text of the original publisher's 3. ed.). San Francisco, Calif.

Lebrun, M. \& Lecoq, J. (2015). Classes inversées: Enseigner et apprendre à l'endroit!. Réseau Canopé.

Lebrun, M., Gilson, C. \& Goffinet, C. (2016). Contribution à une typologie des classes inversées : éléments descriptifs de différents types, configurations pédagogiques et effets. In Education $\&$ Formation. URL: http://revueeducationformation.be.

Mucchielli, R. (2008). Les méthodes actives dans la pédagogie des adultes. ESF, Paris. 
PHILOLOGICAL CLASS. Vol. 26. No. 3

Nissen, E. (2019). Formation hybride en langues : Articuler présentiel et distanciel. In Les Editions Didier. URL : https://hal.archives-ouvertes.fr/hal-01941203.

Perriault, J. (2002). Education et nouvelles technologies: Théorie et pratiques. Nathan.

Rege Colet, N. \& Berthiaume, D. (Eds.). (2013). La pédagogie dans l'enseignement supérieur : repères théoriques et applications pratiques. Bern, Peter Lan.

Soubrié, T. (2008). La difficile articulation du présentiel et de la distance dans le cadre d'un cours hybride en master. In Alsic. No. 11 (2).

Vygotsky, L. S. (1978). Mind in Society: The Development of Higher Psychological Processes. Cambridge, USA, Harvard University.

\section{Данные об авторе}

Родригес Кристин Бланшар - доктор философии, доцент кафедры лингвистических наук факультета иностранных языков, культуры и науки о человеке, университет Клермон Овернь, член лаборатории лингвистических исследований (Клермон-Ферран, Франция).

Адрес: 63037, Франция, Клермон-Ферран, бульвар Жерговья, 29.

E-mail: Christine.Blanchard@uca.fr.

\section{Author's information}

Rodrigues Christine Blanchard - PhD, Associate Professor in the Department of Language Sciences, UFR Lettres, Cultures et Sciences Humaines, Université Clermont Auvergne, member of the Laboratoire de Recherche sur le Langage (Clermont-Ferrand, France). 